Original Research Paper

\title{
Construction and Application of a Statistical Test for Coefficient of Variation on Normal Distributions
}

\author{
Solly Matshonisa Seeletse and Gezani Richman Miyambu \\ Department of Statistics and Operations Research, \\ Sefako Makgatho Health Sciences University, South Africa
}

\author{
Article history \\ Received: 14-07-2017 \\ Revised: 23-10-2017 \\ Accepted: 11-11-2017 \\ Corresponding Author: \\ Solly Matshonisa Seeletse, \\ Department of Statistics and \\ Operations Research, Sefako \\ Makgatho Health Sciences \\ University, South Africa \\ Email: solly.seeletse@smu.ac.za
}

\begin{abstract}
In this study a novel statistical test is derived for the Coefficient of Variation (CV) under normal distributions. This is a newly derived test with value to engineering sciences in aspects of production of accurate items. The $\mathrm{CV}$ can measure the precision of a measuring instrument, among other applications. In order to determine instrument reliability, start by generating measures using the instrument. The $\mathrm{CV}$ is then calculated to determine if the measures generated by the instrument are concentrated around a central point. In use of normal distribution presumption, or approximation, applicable properties of the normal distributions lead to involvement of the chi-square and t-distributions. A CV test is then constructed, and two illustrative examples conclude the discussion.
\end{abstract}

Keywords: Central Limit Theorem, Coefficient of Variation, Law of Large Numbers, Normal Distribution

\section{Introduction}

\section{Background and Definition of the Problem}

Let $X$ be a continuous random variable with mean $\mu$ and variance $\sigma^{2}$. As it is usually difficult to know these parameters, sample values are often used to estimate them (Hinkelmann and Kempthorne 2008; Nicholas 2006). Consider a random sample of $n$ observations from $X$ denoted by $X_{1}, X_{2}, \ldots ., X_{n}$. Let $\bar{X}$ and $S^{2}$ be the sample mean and sample variance. These sample estimates are respective estimators of $\mu$ and $\sigma^{2}$. The square root $\sigma$ of $\sigma^{2}$ is the population standard deviation. Similarly, the square root $S$ of $S^{2}$ is the sample standard deviation.

\section{Review of Existing Literature}

Several authors (Bennett and Briggs 2005; Gelman 2005; 2008; Kleijnen 2000; Reed et al. 2002) define the population $\mathrm{CV}$ as:

$$
V=\frac{\sigma}{\mu}
$$

According to several authors (Armitage 2005; Kleijnen and Sargent 2000; Reed et al. 2002), the CV value provides the precision of any measuring instrument or sampling procedure used. Kleijnen (2000) remarks further that there is no known exact statistical test for testing the coefficient of variation. This remark stimulated interest in developing such a test.

\section{Purpose of the Present Study}

This paper aims to contribute in testing hypotheses that involve $V$.

Consider the gamma function which has the form $\Gamma(k)=\int y^{k-1} e^{-y} d y, k>0$. Before the discussion takes off, two assumptions are made. First, it is assumed that the sample sizes used in this study are large enough to offset the pitfall of lack of representativeness that could result from some small samples. The second assumption is that samples are drawn from populations that are normally distributed.

\section{Formulation of the Hypothesis}

Suppose that experience shows that $X$ has a specified mean $\mu_{0}(\neq 0)$ and variance $\sigma_{0}^{2}(>0)$. Then it is tempting to believe that the general variation in repeated measurements taken from $X$ is given by the $\mathrm{CV}$ :

$v_{0}=\frac{\sigma_{0}}{\mu_{0}}$

To test the assumption that the $\mathrm{CV}$ is $v_{0}$, let a random sample of size $\mathrm{n}$ from $X$ be given. Also, let $\bar{X}$ and $S^{2}$ be the respective sample estimates of $\mu_{0}$ and $\sigma_{0}^{2}$. The aim is to test the null hypothesis $\mathrm{H}_{0}$ given by: 


$$
\mathrm{H}_{0}: v=v_{0}
$$

Denote by $H_{a}$, any alternative of $H_{0}$. The test statistic $V$ is an estimate of $v$, where:

$$
V=\frac{s}{\bar{x}}
$$

\section{Construction of a Hypothesis Test for $V$}

A chi-square distribution derives from the quotient of a standard normal and chi-square distribution. Consider two independent random variables, $Z$ which is standard normal random in distribution and $X_{k}^{2}$, which has a chisquare distributed random variable with $k$ degrees of freedom. Pillai (2016) and Westfall (2013) showed that the random variable $t$ defined in Equation (5) below, has a t-distribution with $k$ degrees of freedom, where:

$$
t=\frac{Z}{\sqrt{\frac{\chi_{k}^{2}}{k}}}
$$

This section uses sampling distributions of known statistics that are associated with $V$ to derive a statistical test given by equation (4). Cox (2006) demonstrates that for any sample size $n$ from a normal distribution, the quantity:

$$
\frac{(n-1) S^{2}}{\sigma_{0}^{2}}
$$

has a $\chi_{n-1}^{2}$ distribution $\bar{X}$ and $\mathrm{S}^{2}$ are independent random variables. By implication, $\bar{X}$ and $S$ are independent random variables.

In the forthcoming discussion, the symbol ' $\sim$ ' shall denote the phrase 'is distributed as'. Recall the random sample $X_{1}, X_{2, \ldots . .,} X_{n}$ from $X$ with mean $\mu_{0}$. Define:

$$
X_{i}^{*}=X_{i}-\mu_{0} \text { for } i=1,2, \ldots, n
$$

Therefore, the mean of $X_{i}^{*}$ is $\mu_{i}^{*}=0$ and variance is $\sigma_{i}^{2^{*}}=\sigma_{0}^{2}$ for all $i=1,2, \ldots, n$.

Tabachnick and Fidell (2007) ascertain that if the sample was drawn from a normal distribution, then for any $n$,

$$
\sqrt{n \frac{\bar{x}^{*}}{\sigma_{0}^{*}}}
$$

has an approximate standard normal distribution. Then using equations (5) and (6), the statistic:

$$
\frac{\sqrt{n \frac{\bar{X}^{*}}{\sigma_{0}^{2}}}}{\sqrt{\frac{(n-1) S^{2}}{\sigma_{0}^{2}} /(n-1)}}=\frac{\sqrt{n \bar{X}^{*}}}{S} \sim \mathrm{t}_{\mathrm{n}-1}
$$

where, $t_{n-1}$ is the t-distribution with n-1 degrees of freedom.

From expansion of (8):

$$
\frac{\sqrt{n \bar{X}^{*}}}{S}=\frac{\sqrt{n \bar{X}-n \mu_{0}}}{S}
$$

Then applying the above equation on (8) leads to:

$\sqrt{\frac{n \bar{X}-n \mu_{0}}{S}} \sim \mathrm{t}_{\mathrm{n}-1}$

Recalling Equation (4):

$$
V=\frac{s}{\bar{X}}
$$

Then, in equation (9) we will be able to conclude and use the equation:

$$
\bar{X}=\frac{S}{V}
$$

Add-on operations from Equations (8) and (9) are:

$$
\begin{aligned}
& \sqrt{n \frac{S}{V}-n \mu_{0}} \sim \mathrm{St}_{\mathrm{n}-1} \\
& \Rightarrow n \frac{S}{V}-n \mu_{0} \sim \mathrm{S}^{2} \mathrm{t}_{\mathrm{n}-1}^{2} \\
& \Rightarrow n \frac{S}{V} \sim n \mu_{0}+\mathrm{S}^{2} \mathrm{t}_{\mathrm{n}-1}^{2}
\end{aligned}
$$

Variate relationships of inverses and transformation of random variables exist with the basic mathematical operations (Forbes et al. 2000). Assuming that these mathematical properties, coupled with the aforesaid manipulations, are permissible with $\sim$, then from equation (9):

$V \sim \frac{\mathrm{n}}{\mathrm{S}\left(n \mu_{0}+S t_{n-1}^{2}\right)}$

The calculated value of $V$ is denoted by $V_{\text {calc }}$, while $t_{m, p}$ shall denote the table value from $\mathrm{t}$-distribution with $m$ degrees of freedom and the probability $p$ on the upper region of the table of t-distributions.

The next discussions use capital letters for denoting the estimators and small letters for denoting estimates. Estimators are statistics and therefore random variables 
with probability distribution. Small letters are used for calculating sample estimates from actual data observed.

\section{Theoretical Construction of Critical Region}

This section is intended to construct the critical region for the test initiated at Equation (3). Consider a random sample for the normal distribution with mean $\mu$. The next formation uses equation (5), which in brief, states the fact that if $Z$, which is distributed as standard normal (denoted by $Z \sim N(0,1)$ ) and $Y \sim X_{n}^{2}$ are independent random variables (Broverman, 2001), then:

$$
T=\frac{Z}{\sqrt{Y / n}} \sim t_{n-1}
$$

Research statisticians (Bless and Kathuria 1993; Wackerly et al. 2007) show that the $t$-test is as follows.

From Equation (10), the test statistic:

$$
T=\sqrt{n \frac{S}{V}-n \mu_{0}} \sim S t_{n-1}
$$

The test procedure follows. The null hypothesis is:

$$
H_{0}: V=v_{0}
$$

\section{Upper Alternative}

Suppose that the alternative hypothesis is:

$$
H_{a}: V>v_{0}
$$

Then, by virtue of $V$ being indirectly proportional with $\mu_{0}$, we reject $H_{0}$ if:

$$
V_{\text {calc }}<\frac{n}{S\left(n \mu_{0}+S^{2} t_{n-1, \alpha}^{2}\right)}
$$

where, $s$ is the estimate of the population standard deviation.

\section{Lower Alternative}

Let:

$$
H_{a}: V<v_{0}
$$

Then, reject $H_{0}$ if:

$$
V_{\text {calc }}>\frac{n}{S\left(n \mu_{0}+S^{2} t_{n-1, \alpha}^{2}\right)}
$$

\section{Two-Sided Alternative}

Lastly, let:

$$
H_{a}: V \neq v_{0}
$$

Then, reject $H_{0}$ if:

$$
V_{\text {calc }}<\frac{n}{S\left(n \mu_{0}+S^{2} t_{n-1, \alpha}^{2}\right)}
$$

Or:

$$
V_{\text {calc }}>\frac{n}{S\left(n \mu_{0}+S^{2} t_{n-1, \alpha}^{2}\right)}
$$

\section{Methodology}

This section basically explains the test procedure. The test approach for the $\mathrm{CV}$ is guided by the following premises:

$$
\begin{aligned}
& H_{0}: V=v_{0} \\
& V_{S} \\
& H_{a}: \text { Not } H_{0}
\end{aligned}
$$

In this case, where $v_{0}$ is specified, but neither $\mu$ nor $\sigma$ are stated, then for the test statistic $V, \mu_{0}$ shall be estimated by using:

$\mu_{0}=\frac{s}{v_{0}}$

Also at Equation (10), for sufficiently large sample size $n, z_{p}$ will be employed in the place of $t_{n-1, p}$ due to use of the approximate normal distribution from the Law of Large Numbers (LLN) and the Central Limit Theorem (CLT) (Berenson et al. 2012; Fischer 2011; Freedman 2005; Kleijnen 2000; Reed et al. 2002). In fact, from LLN and CLT, for large n (or degrees of freedom), the distribution of the t-statistic approaches a standard normal distribution.

\section{Pragmatic Resolution on Critical Regions}

Deficiency of the t-Test

One probable problem in the $t$-test is that the investigator can determine the significance level. Thus, the user can retain or reject the null hypothesis based upon their decision (Mankiewicz 2000). Even though not proper, an analyst can potentially influence the confidence interval in order to achieve the preferred result.

Another concern with the t-test is that the results are precisely truthful only with normal populations (Raju 2005). On the other hand, Rice (2006) counsels that real 
populations are never exactly normal. The techniques for t-tests are fortunately reasonably robust against nonnormality in the population except in the presence of outliers or significant skewness. Larger samples greatly improve the accuracy of the critical values in $t$ distributions when the population is not normal. Dodge (2008) explains that due to the CLT, the sampling distribution of the sample mean from a sufficiently large sample is approximately normally distributed. Also, as the sample size increases, the sample standard deviation converges to the population standard deviation. Sawilowsky (2005) warns analysts to be cautious when applying the t-tests because of hidden influences from influential data. Fay and Proschan (2010) also suggest alternative tests when conditions for use of a t-test are questionable, or the test is likely going to be subjected to misleading effects. In the case of the test in this paper, a squared $t$ has emerged and there are therefore doubts in the direct use of the t-tests. Thus, the t-test is proposed for application only to as far as it relates to other distribution(s).

\section{Approximate Construction}

Let $Z_{1}, Z_{2}, \ldots, Z_{k}$ be normal, identically and independently distributed (iid) with mean 0 and variance 1 , denoted by $Z_{i} \sim$ iid $N(0,1)$ for $i=1,2, \ldots, k$. Then, Bagdonavicius et al. (2011) and Lomax (2007) show that:

$$
X=Z_{1}^{2}+Z_{2}^{2}+\ldots+Z_{k}^{2} \sim \chi_{k}^{2}
$$

This equation is fundamental in relationships of the normal $t-, X^{2}$ and F-distributions (Corder and Foreman, 2014; Jaynes, 2003).

\section{Relationship between $X^{2}$ and F-Distributions}

For $n$ independent observations from a $N\left(\mu, \sigma^{2}\right)$, the sum of the squared standard scores has a chi-square distribution with $n$ degrees of freedom (Bagdonavicius and Nikulin 2011). Chi-squared distribution only depends on degrees of freedom, which in turn depends on sample size $n$. The standard scores are computed using population $\mu$ and $\sigma^{2}$. However, the actual values of $\mu$ and $\sigma^{2}$ are usually not known. When $\mu$ and $\sigma^{2}$ are estimated from the sampled data, the degrees of freedom are less than $n$.

The $F$ distribution is used in tests to compare if two variances are equal (DeGroot and Schervish 2011). The test starts with two independent populations, $Y_{1}$ and $Y_{2}$, each being normally distributed and having equal variances. Then, let $Y_{1} \sim i i d N\left(\mu_{1}, \sigma^{2}\right)$ and $Y_{2} \sim i i d N\left(\mu_{2}\right.$, $\sigma^{2}$, and draw two independent random samples fromeach population, with sample sizes $n_{1}$ from population 1 and $\mathrm{n}_{2}$ from population 2 .

According to Larsen and Marx (2001), in constructing the $F$ distribution using data from each of the two samples, estimate $\sigma^{2}$ using the pooled variance from sample variances $s_{1}^{2}$ and $s_{2}^{2}$. Both $S_{1}^{2}$ and $S_{2}^{2}$ are random variables. Furthermore, their ratio is a random variable:

$$
F=\frac{\text { estimate of } \sigma_{1}^{2}}{\text { estimate of } \sigma_{2}^{2}}=\frac{s_{1}^{2}}{s_{2}^{2}}
$$
thus:

This can further be presented mathematically and

$$
F=\frac{\chi_{n_{1}-1}^{2} /\left(n_{1}-1\right)}{\chi_{n_{2}-1}^{2} /\left(n_{2}-1\right)}
$$

Therefore, the random variable $F$ defined from two independent chi-square variables has an F-distribution.

\section{Relationship between $t$ - and F-Distributions}

The procedures described are mathematically valid, as they satisfy mathematical principles. However, reality, according to Abramowitz and Stegun (2012), is that $t_{k}^{2}=F_{1, k}$. This means that the squared t-distribution with $k$ degrees of freedom is the same as the F-distribution with 1 and $k$ degrees of freedom.

\section{F-Test}

An $F$-test is a statistical test with the test statistic that has an F-distribution when the null hypothesis is true (Maddala and Lahiri 2009). It is used to compare statistical models that have been fitted to a dataset to identify the model with best fit to the study population. As shown, the F-distribution is a ratio of two independent chi-square random variables that are divided by the respective degrees of freedom.

The F-test is developed to test equality of two population variances (Bulmer 2012). It does this by comparing the ratio of two variances. Thus, if the variances are equal, the ratio of the variances equals 1 . If the null hypothesis is true, then the F-test statistic can be simplified. The ratio of sample variances is the test statistic used. If the null hypothesis is false, then the decision is to reject the null hypothesis that the ratio is equal to 1 and thus the assumption of equality.

The F-tables only give levels of significance for right tailed tests. Since the F-distribution is not symmetric, and there are no negative values, we do not simply take the opposite of the right critical value to obtain the left critical value (Carlberg 2014). The way to find a left critical value is to reverse the degrees of freedom, find the right critical value, and then take the reciprocal of this value.

\section{Formation of F-Test}

The $F$ is formed by chi-square (Sawilowsky 2002) and therefore many of the chi-square properties carry over to the F-distribution. In conducting the F-tests, then 
the following conditions are valid (Ryabko et al. 2004): The F-values are non-negative. The F-distribution is non-symmetric. Its mean is about 1 . There are two independent degrees of freedom, where one is for the numerator and the other is for the denominator. Also, there are many different F-distributions, one for each pair of degrees of freedom.

The approach in using the F-distribution is to avoid left critical values (Bagdonavicius and Nikulin 2002). Generally, the left critical values are difficult to calculate. As a result they are often avoided. A strategy is to influence the F-test towards a right tailed test by assigning the sample with the large variance in the numerator and the smaller variance in the denominator. Even though it does not matter which sample has the larger sample size, it matters that the sample having the larger variance is placed in the numerator.

In developing the F-tests, the following assumptions are necessary in the use of the F-distribution (Cacoullos 1965; Fadem 2012). The larger variance should always be placed in the numerator. The test statistic is:

$$
F=s_{1}^{2} / s_{2}^{2} \text { where } s_{1}^{2}>s_{2}^{2}
$$

In the case where a two-sided alternative hypothesis would have been appropriate, the approach should be to divide the significance level (or alpha) by 2 and then obtain the right critical value (Galecki and Burzykowski 2013). When the degrees of freedom are not given in the table, then the value with the larger critical value should be chosen. This is the smaller degrees of freedom and reduces the likelihood of type I error.

The study populations providing the samples have to be normal (or approximately normal at least) and the sampling must be conducted using a random sampling method. These random samples must be independent.

\section{Numerical Illustrations}

\section{Car Crash Prices Data}

The following scores in Table 1 are the prices in ZAR1000's of accident damaged cars of the same model at Denver Company, Johannesburg (The Star Classified 1998:4). Assume that before the accidents, all these cars were of equal value.

Suppose further that if prices of these cars are identical, the $\mathrm{CV}$ is expected to be at most $10 \%$. The question to be addressed is whether the damages caused by the accidents on these cars are significantly dissimilar.

Table 1: Prices of damaged cars at Denver Co

\begin{tabular}{llllllllllll}
\hline 7.5 & 8 & 11 & 13 & 14 & 9.75 & 19.5 & 7 & 13 & 9.5 & 17.5 & 9 \\
15 & 8 & 14 & 8.5 & 12 & 9 & 7 & 8 & 8.5 & 12.5 & 7 & 4.5 \\
6 & 7.5 & 8.5 & 13.5 & 15 & 24 & 14 & 12 & 29 & & & \\
\hline
\end{tabular}

In addressing this question, the information given could be used to determine if $\mathrm{CV}$ of at most 0.1 is an acceptable assumption. The answer is deduced in the following set of operations:

(1) $H_{0}: V \leq 0.1$ vs $H_{0}: V>0.1$

(2) Level of significance, $\alpha=0.1$

(3) $V_{\text {calc }}=\frac{s}{\bar{x}}=\frac{5.217408759784}{11.583333333333}=0.4504$

(4) From equation (15), reject $\mathrm{H}_{0}$ if:

$$
V_{c a l c}<V_{c r i t}=\frac{n}{S\left(n \mu_{0}+S^{2} t_{n-1, \alpha}^{2}\right)} .
$$

Now, $t_{n-1, \alpha}=t_{32,0.1}=1.3086, \frac{\mu_{0}}{\sigma_{0}}=\frac{1}{V}=10$ and $n=33$.

Then the test statistic is:

$$
\begin{aligned}
V_{\text {crit }} & =\frac{n}{S\left(n \mu_{0}+S^{2} t^{2}{ }_{n-1, \alpha}\right)} \\
& =\frac{33}{5.2174\left(33 \times 10 \times 5.2174+5.2174^{2} \times 1.3086^{2}\right)} \\
& =0.0036
\end{aligned}
$$

Since $V_{\text {calc }}>V$, then $\mathrm{H}_{0}$ should not be rejected at the $10 \%$ significance level. It can be decided that the variance of the prices of these cars is not significantly large compared to the mean. Therefore the damages to the cars were not expressively different.

\section{Examination Data}

Howell (2002) provides a possibility to test under the following conditions. Consider a teaching professor in the Department of Psychology at a known University who teaches a group of students with learning disabilities. She finds it rewarding if after each test has been written she can determine if the test was easy or difficult. According to her, this has positive implications for her teaching approach of the subject as well as for assessment purposes. One of her discoveries is that if the student marks are in the ratio of standard deviation to mean of $3: 10$ or more, it is impossible to determine the level of examination difficulty. Recently, she examined her class of 700 students. The marks (in percentages) produced an average of 53 with a standard deviation of 14.143. The analysis should establish if the professor's teaching can benefit from the latest test.

In response to the above question, it is known that the professor can benefit upon determining if the test was easy or difficult. The hypothesis that the ratio of standard deviation to mean is $3: 10$ or more is made, which is to assume that she will not benefit from the test. A formal test of hypothesis is made. The professor cannot say if the test was easy or difficult if: 


$$
H_{0}: V \geq 0.3
$$

This is tested against the alternative hypothesis that:

$$
H_{a}: V<0.3
$$

The alternative hypothesis implies that the professor can determine if the test was easy or difficult.

Given that $n=700, \bar{x}=53$ and $s=14.143$, then the test is as follows:

(1) $H_{0}: V \geq 0.3$ vs $H_{a}: V<0.3$

(2) Choose $a=0.05$

(3) $\mathrm{V}_{\text {calc }}=\frac{\mathrm{s}}{\bar{x}}=\frac{14.143}{53}=0.26685$

(4) From (17), reject $H_{0}$ if:

$$
V_{\text {calc }}>V_{c r i t}=\frac{n}{S\left(n \mu_{0}+S^{2} t_{n-1, a}^{2}\right)}
$$

Now, $\mathrm{t}_{\mathrm{n}-1, \alpha} \cong \mathrm{z}_{0.05}=1.645, \frac{\mu_{0}}{\mathrm{~s}}=\frac{1}{\mathrm{v}_{0}}=\frac{10}{3}$, the test statistic is:

$$
\begin{aligned}
& V_{\text {crit }}=\frac{n}{S\left(n \mu_{0}+S^{2} t_{n-1, \alpha}^{2}\right)} \\
& =\frac{700}{14.143\left(700 \times(10 / 3) \times 14.143+14.143^{2} \times 1.645^{2}\right)}=0.0015 .
\end{aligned}
$$

Since $V_{\text {calc }}>V_{\text {crit }}$, then $H_{0}$ should be rejected. It seems that the level of difficulty of the test can be ascertained. It is therefore acceptable to believe that at the $5 \%$ significance level, the professor can benefit from this test for her teaching, or from the information available.

\section{Conclusion}

The assertion by Kleijnen and other researchers that there are no known exact statistical tests for the CV stimulated this paper. Auspiciously, the gap identified has been addressed with the work in this paper. The paper contributes to knowledge by increasing the tools for analysis, which in this study is a statistical test for $\mathrm{CV}$ under normal distributions. These tests also envelop approximate normality, and extend to the CLT and LLN.

\section{Acknowledgement}

The authors are grateful to the support from the Department of Statistics and Operations Research of Sefako Makgatho Health Sciences University.

\section{Funding Information}

The Department of Statistics and Operations Research funded the manuscript activities.

\section{Authors' Contributions}

Gezani Richman Miyambu: Miyambu provided the mathematical methods for the test statistics and wrote some sections of the paper.

Solly Matshonisa Seeletse: Seeletse selected the case studies used in the numerical illustrations and performed the calculations.

\section{Ethics}

The study benefitted from secondary data and the necessary acknowledgements have been provided by means of references.

\section{References}

Abramowitz, M. and I.A. Stegun, 2012. Handbook of Mathematical Functions. New York: Courier Corporation, ISBN-10: 0486158241, pp: 1046.

Armitage, C.J., 2005. Can the theory of planned behavior predict the maintenance of physical activity? Health Psychol., 24: 235-245. DOI: 10.1037\%2F0278-6133.24.3.235

Bagdonavicius, V. and M.S. Nikulin, 2002. Accelerated LIFE Models: Modeling and Statistical Analysis. Taylor and Francis, ISBN-10: 1584881860, pp: 334.

Bagdonavicius, V. and M.S. Nikulin, 2011. Chi-squared goodness-of-fit test for right censored data. Int. J. Applied Math. Statistics, 24: 30-50.

Bagdonavicius, V., J. Kruopis and M. Nikulin, 2011. Non-parametric tests for censored data. Bordeaux, France: Iste.

Bennett, J. and W. Briggs, 2005. Using and Understanding Mathematics: A Quantitative Reasoning Approach. 3rd Edn., Pearson Addison Wesley, ISBN-10: 0321227735, pp: 742.

Berenson, M.L., D.M. Levine and K.A. Szabat, 2012. Basic Business Statistics: Concepts and Applications, 13th Edn., Pearson Higher Education AU, ISBN-10: 1486002447, pp: 676.

Bless, C. and R. Kathuria, 1993. Fundamentals of Social Statistics: An African Perspective. Juta and Co, ISBN-10: 0702129402, pp: 367.

Broverman, S.A., 2001. Actex study manual, Course 1, Examination of the Society of Actuaries, Exam 1 of the Casualty Actuarial Society. Winsted, CT: Actex Publications, ISBN-10: 1566984017.

Bulmer, M.G., 2012. Principles of Statistics. Edinburgh: Oliver and Boyd. Courier Corporation, ISBN-10: 0486135209, pp: 256.

Cacoullos, T., 1965. A relation between $\mathrm{t}$ and $\mathrm{F}$ distributions. J. Am. Statistical Assoc., 60: 528-531. DOI: $10.2307 / 2282687$

Carlberg, C., 2014. Statistical Analysis: Microsoft Excell 2013. Que Publishing, ISBN-10: 0133823997, pp: 512. 
Corder, G.W. and D.I. Foreman, 2014. Nonparametric Statistics: A Step-by-Step Approach. Wiley, New York, ISBN-10: 1118840313, pp: 267.

Cox, D.R., 2006. Principles of statistical inference. New York: Cambridge University Press.

DeGroot, M.H. and M.J. Schervish, 2011. Probability and Statistics, 4th Edn., New York: AddisonWesley. ISBN-10: 0321831020, pp: 912.

Dodge, Y., 2008. The Concise Encyclopedia of Statistics. New York: Springer Science and Business Media. ISBN-10: 0387317422, pp: 622.

Fadem, B., 2012. High-yield Behavioral Science (highyield series). Hagerstwon, MD: Lippincott Williams and Wilkins. ISBN-10: 1451130309, pp: 144.

Fay, M.P. and M.A. Proschan, 2010. Wilcoxon-Mannwhitney or t-test? On assumption for hypothesis tests and multiple interpretations of decision rules. Statist. Surv., 4: 1-39.

Fischer, H., 2011. A history of the central Limit theorem: From classical to modern probability theory, sources and studies in the history of mathematics and physical sciences. New York: Springer.

Forbes, C., M. Evans, N. Hastings and B. Peacock, 2000. Statistical Distributions, 4th Edn., Hoboken, N.J.: Wiley. ISBN-10: 0471371246, pp: 248.

Freedman, D.A., 2005. Statistical Models: Theory and Practice. 1st Edn., Cambridge University Press. ISBN-10: 0521854830, pp: 414.

Galecki, A. and T. Burzykowski, 2013. Linear mixedeffects models using R: A Step-by-Step Approach. 1st Edn., New York: Springer Science and Business Media. ISBN-10: 1461439000, pp: 542.

Gelman, A., 2005a. Analysis of variance: Why it is more important than ever. Annals Statist., 33: 1-31.

Gelman, A., 2008. Analysis of variance. The new Palgrave dictionary of Economics, 2nd Edn., Basingstoke, Hampshire New York: Palgrave Macmillan.

Hinkelmann, K. and O. Kempthorne, 2008. Design and Analysis of Experiments. I and II, 2nd Edn., London: Wiley.

Howell, D.C., 2002. Statistical Methods for Psychology, 5th Edn., Pacific Grove, CA: Duxbury/Thomson Learning. ISBN-10: 053437770X, pp: 802.

Jaynes, E.T., 2003. Probability Theory: The Logic of Science. 1st Edn., Cambridge University Press, ISBN-10: 0521592712 pp: 727.

Kleijnen, J.P.C. and R.G. Sargentb, 2000. A methodology for fitting and validating met models in simulation. Eur. J. Oper. Res., 120: 14-29. DOI: $10.1016 / \mathrm{S} 0377-2217(98) 00392-0$

Kleijnen, J.P.C., 2000. Strategic Directions in Verification, Validation and Accreditation Research: A personal view. Tilburg University.
Larsen, R.J. and M.L. Marx, 2001. An Introduction to Mathematical Statistics and its Applications, 3rd Edn., Upper Saddle River, NJ: Prentice-Hall. ISBN-10: 0139223037, pp: 790.

Lomax, R.G., 2007. Statistical Concepts: A Second Course. 1st Edn., New York: Lawrence Erlbaum Associates. ISBN-10: 0805858504, pp: 266.

Maddala, G.S. and K. Lahiri, 2009. Introduction to Econometrics, 4th Edn., Chichester, Wiley. ISBN-10: 0470015128, pp: 654.

Mankiewicz, R., 2000. The Story of Mathematics. 1st Edn., Princeton, NJ: Princeton University Press. pp: 192.

Nicholas, J., 2006. Descriptive statistics. Mathematics Learning Centre, University of Sydney, New South Wales.

Pillai, N.S., 2016. An unexpected encounter with cauchy and lévy. Ann. Statist., 44: 2089-2097.

Raju, T.N., 2005. William Sealy Gosset and William a Silverman: Two "students" of science. Pediatrics, 116: $732-735$.

Reed, J.F., F. Lynn and B.D. Meade, 2002. Use of coefficient of variation in assessing variability of quantitative assays. Clin Diagn Lab Immunol., 9: 1235-1239.

DOI: 10.1128/CDLI.9.6.1235-1239.2002

Rice, J.A., 2006. Mathematical Statistics and Data Analysis, 3rd Edn., New York: Duxbury Advanced. ISBN-10: 0534399428, pp: 688.

Ryabko, B.Y., V.S. Stognienko and Y.I. Shokin, 2004. A new test for randomness and its application to some cryptographic problems. J. Statist. Planning Inference, 123: 365-376. DOI: $10.1016 / \mathrm{S} 0378-3758(03) 00149-6$

Sawilowsky, S., 2002. Fermat, Schubert, Einstein and Behrens-Fischer: The probable difference between two means when $\sigma^{1} \neq \sigma^{2}$. J. Modern Applied Statist. Methods, 1: 461-472. DOI: $10.22237 / \mathrm{jmasm} / 1036109940$

Sawilowsky, S.S., 2005. Misconceptions leading to choosing the $\mathrm{t}$ test over the Wil Coxon-MannWhitney test for shift in location parameter. J. Mod. Applied Statist. Methods, 4: 598-600. DOI: $10.22237 / \mathrm{jmasm} / 1130804700$

Tabachnick, B.G. and L.S. Fidell, 2007. Using Multivariate Statistics, 5th Edn., Pearson. ISBN-10: 0205465250, pp: 980.

The Star Classified, 1998. Denver Company, Johannesburg.

Wackerly, D., W. Mendenhall and R.L. Scheaffer, 2007. Mathematical Statistics with Applications, 7th Edn., Duxbury Press, ISBN-10: 0495427829

Westfall, P.H., 2013. Understanding Advanced Statistical Methods. 1st Edn., Boca Raton, FL: CRC Press. ISBN-10: 1466512105, pp: 569. 